\title{
Aspects of Oxidative Metabolism in Schizophrenia
}

\author{
R. M. MARCHBANKS, J. MULCRONE and S. A. WHATLEY
}

Investigations of the biological phenomenology and aetiology of schizophrenia have produced a complex pattern which defies unitary theories. It is not surprising that within this framework several competing theories are equally viable and few can be totally dismissed. One area which has received little attention has been the area of oxidative metabolism (the site of which is the mitochondrion), presumably because it has no obvious association with either the classical pharmacological systems generally assumed to underlie schizophrenic biology or with structural biology. The association of mitochondrial function with human disease has, however, only 35 years after the demonstration of mitochondrial involvement in myopathy, become a rapidly expanding field.

Changes in mitochondrial function are now implicated in over 100 diseases, usually associated with mutations in the mitochondrial genome. Primary mutation in the mitochondrial genome generally affects tissues with a large energy demand, such as muscle and brain, and result in specific clinical syndromes some of which are discussed in detail below. In addition, however, mitochondrial mutations and impaired oxidative metabolism are also observed associated with some common age-related illnesses, probably as secondary phenomena. These conditions include the ageing process itself as well as specific central nervous system disorders such as Parkinson's disease, Alzheimer's disease, amyotrophic lateral sclerosis, and Huntingdon's disease. The exact relationship between these mitochondrial changes and these illnesses is still under debate, but this serves to illustrate the growing influence of ideas regarding oxidative phosphorylation in the debate about human disease.

In the phenomenology of schizophrenia two main biological clues stand out and yet their exact role remains frustratingly elusive.

First, epidemiological studies have demonstrated a genetic component in the liability to develop schizophrenia. Despite segregation and linkage analysis, however, neither the genes involved nor the mode of transmission have been established. Studies of monozygotic twins have demonstrated an additional environmental component in the development of schizophrenia. This has led to the development of multifactorial models in which a polygenic inheritance component of $68 \%$ and an environmental component of $32 \%$ are compatible with European family and twin data.

Second, one of the main theories of schizophrenia aetiology has been the dopamine hypothesis which proposes relative or absolute dopaminergic hyperactivity in the brain due to a combination of increased neurotransmitter production or release at terminals, or increased receptor sensitivity or reduced activity of endogenous dopamine antagonists. A range of biochemical studies however has failed to provide consistent evidence for this theory. In addition, genetic linkage studies in families have uniformly failed to find evidence of linkage of dopamine receptors $D 1,2,3,4$ or 5 to schizophrenia in multiply-affected pedigrees (Coon et al, 1993; Su et $a l, 1993)$. These results probably rule out a major primary contribution to schizophrenic aetiology from mutant dopamine receptor populations, although less frequent or more subtle contributions are possible. The study of such contributions by population analysis has produced either no or mixed evidence for associations between either D1 or D2 and D3 receptor variants and schizophrenia. Direct sequencing strategies have also not been able to uncover dopamine receptor mutations in schizophrenics (Gejman et al, 1994).

Schizophrenia is regarded as a functional psychosis in that it is thought to arise mainly as a result of the disorderly activity of neurons rather than structural or pathological alterations of the brain. Yet despite extensive research into the biochemical basis of schizophrenia a critical dysfunction has not yet been shown to be specific to the clinical condition. To date the results of a number of divergent investigations have implicated altered brain metabolism in schizophrenia. Theories based on these observations, however, have received little interest or support. The results of our own research have led us to examine the possible role of altered oxidative metabolism in schizophrenia. This paper attempts to reappraise some aspects of the phenomenology of schizophrenia and in combination with our own findings propose some working hypotheses.

\section{Positron Emission Scanning}

Positron Emission Tomography (PET) has made a considerable contribution to the biology of 
schizophrenia because it enables the changes in a metabolic variable to be measured in real time in situ under controlled behavioural circumstances. In its application to oxidative metabolism the short lived positron emitting ${ }^{18} \mathrm{~F}$-2-fluoro-2-deoxy-D-glucose is administered to the patient. 2-deoxy glucose acting here as an analogue of glucose is rapidly taken up by tissues undergoing active oxidative metabolism but after phosphorylation cannot then be further metabolised so that it accumulates at the position of uptake. The regions of accumulation can then be detected by the positron emission resulting from the isotopic decay of the ${ }^{18} \mathrm{~F}$ attached to the deoxyphosphorylglucose molecule. Using the standard procedures of tomography the source of emission can be located with some precision in whole tissues because the positron (like the X-ray) is not appreciably scattered.

It is important to emphasise that this elegant technique reveals directly the site of changed glycolysis rather than glucose metabolism in the wider sense of oxygen utilisation and energy supply. In fact the rates of glycolysis and oxygen utilisation are tightly coupled by means of allosteric control of the enzyme 6-phosphofructo-1-kinase. The activity of this enzyme controls the rate of glycolysis and it is upregulated by the substrates of cytochrome oxidase (see below), ADP and inorganic phosphate, and downregulated by its product ATP. In addition, it is downregulated by citrate and hydrogen ions and cyclic AMP. A lowered uptake of deoxyglucose therefore implies a sufficient ratio of ATP/ADP.P for cellular needs or an overabundance of either citrate, hydrogen ions, cyclic AMP or any combination of them. ATP is the ubiquitous energy 'currency' of nerve cells and the ratio ATP/ADP.P. directly controls oxygen uptake in mitochondria by cytochrome oxidase.

\section{Hypofrontality}

Studies on aerobic metabolism in schizophrenia could be said to have started with those of Takahashi who observed a reduced oxygen uptake in brain biopsies from schizophrenics (Takahashi, 1954). The original studies on deoxyglucose uptake by Ingvar and his colleagues in 1982 had been stimulated by their earlier finding that the cerebral blood flow measured by the xenon clearance technique was lower in the frontal cortex of chronic schizophrenics than in normals (Franzen \& Ingvar, 1975). The application of the technique to schizophrenics in defined behavioural situations was first undertaken by Cohen and his colleagues (Cohen et al, 1987). They found that glucose metabolic rates in the left and right pre-frontal cortex of drug-free schizophrenics were not elevated as much as normals while performing an auditory discrimination task even though their performance was as good as the normals. Schizophrenics on medication did not show this phenomenon. The effect of neuroleptic medication on glucose metabolic rates appears to differ depending on the brain region but the generalisation holds that lowered glucose utilisation in the frontal cortex (hypofrontality) is not an effect of neuroleptic medication (Cohen et al, 1988). These results were in essence confirmed by Buchsbaum et al (1990) in patients who had been denied psychoactive medication for 31 days and who were matched with normals having the same right-handed manual preference. A visual discrimination task was given which raised the glucose utilisation levels in the right frontal and right temporoparietal regions. Schizophrenic patients showed in comparison to normals a much attenuated utilisation response in the cortex and it was noted that the anatomical pattern of the response differed from normals. Although in normals there was a positive correlation between task performance and glucose metabolism, this was reversed in schizophrenics.

The general result can be described as a reduction in unmedicated schizophrenics of the task-elicited glucose utilisation in the frontal cortex which is not related to deficiencies in performance. Although this effect is not caused by currently administered medication there is unfortunately no consensus as to what effects psychoactive drugs do have on hypofrontality; for a discussion see Szechtman et al (1988). Hypofrontality could be caused by reduced requirement of the cells (neurons or glia) of the frontal cortex for metabolic energy or an abnormality in the components of the regulatory system in which cytochrome oxidase plays a pivotal role.

\section{Effects of medication}

Antagonism of brain dopamine receptors is considered to be involved in the therapeutic effects of neuroleptic drugs (for review see Sunhara et al, 1993). Although the site of primary action of therapeutic drugs is fairly clear, exact molecular mechanisms which underlie the response to neuroleptics are poorly understood. In particular, while blockade of brain dopamine receptors occurs after acute administration of these drugs, the antipsychotic effects are noted 2-3 weeks after repeated administration (Johnstone et al, 1978). Longer term processes secondary to dopamine receptor antagonism may thus be responsible for the drug action, and it is highly likely that these will involve changes in gene expression patterns in the brain. 
Explanations for therapeutic delay have included adaptive changes in receptor systems, and indeed changes in dopamine metabolism, receptor number and firing rates have been noted in animal models during repeated administration. These occur over time periods which are consistent with therapeutic action, and it has been suggested that adaptive changes are themselves associated with clinical response (Chiodo \& Bunney, 1983). While these particular adaptive theories may be criticised on both theoretical and pharmacological grounds, the idea of long-term changes occurring in brain as a result of dopamine blockade is consistent with the known properties of the dopamine receptors, which are a family of metabotropic receptors linked to second messenger systems via stimulation or inhibition of cyclic AMP production. Stimulation of these receptors therefore is not linked directly to ion channel gating but to modulation of firing via intracellular signalling. Cyclic AMP itself is a ubiquitous signalling molecule with pleiotropic effects in the cell. cAMP pathways include both shortterm events via the membrane and long-term actions via modulation of gene transcription. In addition, however, cyclic AMP is classically a modulator of metabolic activity. For instance, cyclic AMP inhibits glycolysis by stimulating a protein kinase that modifies 6-phosphofructose-1-kinase itself.

Several studies have demonstrated effects of antipsychotic drugs on gene expression in the brain, but most of these have concentrated on either dopamine receptors or other neuromodulatory systems such as neurotensin. However, systems known to be involved in the regulation of gene expression, the immediate early genes have also been studied, and found to be regulated themselves by antipsychotic treatment. This supports the idea that dopamine blockade results in the changes in gene expression in the brain probably through second messenger pathways.

Although there is little information about the end systems affected by dopamine blockade, recent studies in our laboratory have suggested that up to $0.5 \%$ of the total genes expressed in the forebrain are regulated specifically by the therapeutically active (cis-) isomer of flupenthixol. In addition, $25 \%$ of this population of genes are mitochondrially derived, including subunits I and II of cytochrome oxidase which are downregulated (Whatley et al, unpublished observations). This indicates quite widespread effects by this drug on brain gene expression, a major aspect of which is regulation of mitochondrial genes.

\section{Gender differences}

The lifetime risk for schizophrenia is equal for both sexes but the average age of maximal onset is 3-4 years earlier for men compared to women who characteristically have a less severe clinical course of the illness. In addition, within the female population a second, smaller onset peak is consistently observed around the 45-49 year age group. These sexdifferences have resulted in the theory that either oestradiol increases the vulnerability threshold for schizophrenia which is lowered again during menopause or that testosterone reduces the vulnerability threshold. In addition, the increase in affective psychosis observed in females after childbirth is thought to be caused by the sharp fall in circulating oestrogen post-partum. It is thought that these processes are mediated by the dopaminergic system because oestradiol but not testosterone reduced the behavioural changes induced by haloperidol, indicating a downward regulation of dopamine transmission (Hafner et al, 1991).

However, many studies have also shown that gonadal hormones affect mitochondrial function, for example reduced oestrogen has been shown to decrease oxidative phosphorylation and cytochrome oxidase activity in rat muscle (Roth et al, 1989) and in a pituitary cell line oestradiol induced the expression of the mRNA coding for subunit II of cytochrome oxidase (Van-Itallie \& Dannies, 1988). Similarly, an examination of gene expression in ovariectomised rat hypothalamus following oestrogen stimulation resulted in the isolation of an oestrogeninduced clone that corresponded to cytochrome oxidase II (Bettini \& Maggi, 1992). Therefore, it is not implausible to suppose that the sex hormonemediated differences in schizophrenia are mediated by a direct effect on the expression of mitochondrial genes.

\section{Mitochondrial involvement in neuropsychiatric illness}

In recent years a number of mutations in mitochondrial DNA have been identified which are associated with defects in oxidative phosphorylation, and appear to affect tissues in which oxidative phosphorylation is important, mainly muscle, brain, pancreas, kidney and liver. While associated with accumulations of abnormal mitochondria in muscle fibres which show as 'ragged red fibres' on staining, these mitochondrial myopathies represent a clinically diverse group of illnesses which include blindness, ataxia, seizures, deafness, stroke-like episodes, dementia, movement disorders and weakness. Amino acid substitutions in mitochondrial genes are known to result in Leber's optic neuropathy as well as neurogenic muscle weakness, ataxia and retinitis pigmentosa (NARP). A further group of mutations 
affecting the biosynthetic machinery of the mitochondrion are known, most of which involve tRNA mutations. These are known to result in myoclonic epilepsy and ragged red fibre disease (MERRF), the most severely affected family members having pronounced aberrations in brain electrophysiology, neurosensory hearing loss, myoclonus and dementia, and the condition of mitochondrial encephalomyopathy, lactic acidosis and strokelike symptoms (MELAS). Finally, insertion and deletion mutations of mitochondria are known to produce ocular myopathy and Pearson syndrome, which in their most severe forms include ataxia, elevated CSF protein, and dementia. While primarily resulting in neurodegenerative conditions, there has been one report of a case of mitochondrial encephalomyopathy associated with schizophrenic psychosis (Yamazaki et al, 1991). Associations have also been reported between anti-P autoantibodies which react with a mitochondrial replication protein and psychotic symptoms in SLE patients (Schneebaum et al, 1991). These data indicate that some of the signs and symptomatology of schizophrenia accompany known mitochondrial abnormalities.

\section{Genetic consequences of mitochondrial involvement}

One unique feature of mitochondrial genes is that they are inherited maternally, the direct consequence of the fact that the egg harbours several thousand mtDNAs whereas the sperm has only a few hundred. The mitochondrion, however, is only semi-autonomous, since a large proportion of mitochondrial proteins as well as factors required for transcription and translation of mitochondrial genes and replication of the mitochondrial genome are derived from nuclear DNA. The combination of these considerations, the known high mutation rates for mitochondrial DNA and existence of different molecular variants of mitochondria in the same cell (heteroplasmy) may therefore produce fairly complex patterns of inheritance for mitochondrial phenotype.

This is borne out in practice in the case of the encephalomyopathies (for review, see Wallace, 1992), where inheritance patterns are not always clear cut. The great majority of mtDNA deletion syndromes are spontaneous with no family history. Even where mitochondrial inheritance is established, there may not be maternal inheritance through multiple generations due to replicative segregation of mitochondria through generations and threshold expression effects. In addition, mitochondrial abnormalities are known which arise from mutations in genomic DNA. In practice mitochondrial abnormalities are identified by mitochondrial morphology or obvious changes in the oxidative phosphorylation characteristics of tissues such as the brain and muscle combined with genetic data. It is evident, however, that a whole class of mitochondrial syndromes may occur through gain rather than loss of function, and which may also arise from complex interactions between nuclear and mitochondrial genomes.

In the case of schizophrenia, the concept of the 'Schizophrenogenic mother' has been popular in many psychodynamic theories of schizophrenia. Genetic studies themselves have indicated that schizophrenic probands are more likely to have inherited the illness from their mother (Wolyniec et al, 1992). This is generally thought to be due to the earlier age of marriage and the later onset of illness in women compared to men. The post hoc correction of transmission rates is, however, based on generalised assumptions of rates of fecundity which are not verifiable in the populations studied. Theoretical modelling of mitochondrial inheritance has been performed by others (Schork \& Guo, 1993), producing pedigrees which are not unlike many of the published pedigrees which are multiply affected for schizophrenia.

\section{Cytochrome oxidase and the brain}

Cytochrome oxidase (COX) is the pivotal enzyme of aerobic metabolism. All living species that use oxidative metabolism as the energy source for life contain the enzyme although there are considerable inter species differences in subunit composition. The enzyme is present only in mitochondria, and transfers electrons from reduced cytochrome $\mathrm{c}$ to molecular oxygen and protons, during which it creates a proton gradient whose energy is utilised by the mitochondrial $F_{1}$ ATP-ase to convert inorganic phosphate and adenosine diphosphate to adenosine triphosphate (ATP). The three catalytic sequences of COX that carry out the electron transfer and proton gradient formation are highly conserved and coded for by mitochondrial DNA as is the $F_{1}$ ATP-ase. In addition, there are a variable number of nuclear coded subunits (10 in the human) whose function is thought to be connected with the control and perhaps the expression of the enzyme. Several of the genes coding for the nuclear subunits have been cloned and their sequences found to be distributed among various chromosomes. Oxygen uptake in cells is invariably much less than the capacity of the enzyme as measured biochemically indicating intensive cellular regulation. The amount of cytochrome oxidase in mitochondria varies according to the tissue source, those that are aerobically active having the most. 
ATP is the universally accepted energy 'currency' of cells. Neuronal function is dependent on supply of ATP. Synaptic transmitters such as acetylcholine, glutamate and so forth require ATP for their synthesis so that any deficiencies in supply or failure in the control of supply will have an immediate effect on the information processing function of the brain. Deficiencies in the supply of ATP are not necessarily the only results of malfunctioning COX. The enzyme adds single electrons serially to diatomic oxygen so that bound species corresponding to superoxide, peroxide and hydroxyl radical are formed (review: Nacqui $e t a l, 1986)$. All of these species are toxic to cells, hydroxyl radical extremely so. Mutations to the enzyme that caused facile release of any of these species would therefore be pathologically significant.

\section{COX mRNA changes in schizophrenia}

Since the underlying biochemical basis of schizophrenia has yet to be established and linkage analysis has failed to identify chromosomal regions of interest it seemed sensible to approach the problem by attempting to identify those genes whose expression is altered in schizophrenia. Gene expression in the frontal cortex of schizophrenics was examined by using a sequential differential screening protocol that allows a comparative analysis with normal tissue (Mulcrone \& Whatley, 1993). This procedure allowed a numerical comparison of gene expression between the post-mortem frontal cortices of several normal and schizophrenic patients that could then be statistically evaluated. Five differentially expressed cDNAs were isolated whose identification by sequence showed that they were mitochondrially encoded. Three of the cDNAs demonstrating reduced expression in schizophrenic compared to normal tissue were all derived from mitochondrial (mt) 16s rRNA, while of the two displaying an increase in abundance in the schizophrenic compared to control tissue one corresponded to mt 12s rRNA and the other encoded a partial sequence of cytochrome oxidase subunit II (Mulcrone et al, 1995). Furthermore, elevated levels of cytochrome oxidase II mRNA in the frontal cortex of schizophrenic compared to control was confirmed in a larger sample set utilising a quantitative RNA hybridisation procedure.

Interestingly, in this study two of the schizophrenic frontal cortex samples were obtained from patients who were not medicated at the time of death. In these two samples the expression of cytochrome oxidase subunit II was even more increased than in the medicated schizophrenics. Although the results were not available to us at the time this finding is in accord with our subsequent observations on the effects of cis-flupenthixol in reducing the expression of subunits I and II cytochrome oxidase in experimental animals. We have studied the enzymic activity of cytochrome oxidase in frontal cortex from normals and schizophrenics and find, as might be expected considering the centrality of the enzyme to metabolic processes, that there is no significant difference in overall COX activity between the two groups; however, there appear to be differences in the sensitivity of the enzyme to azide inhibition in schizophrenics.

\section{Conclusions}

We propose that the results of our molecular analysis of schizophrenia indicate the involvement of altered mitochondrial function in the symptomatology and possibly the aetiology of the disorder. Among the several mitochondrial components the subunits I and II of cytochrome oxidase suggest themselves because of the sensitivity of their expression to dopaminergic blockade and upregulation by oestrogen. One might speculate that a small proportion of the total cytochrome oxidase having a mutation in this region might so disturb the delicately balanced control mechanisms as to cause the hypofrontality seen by PET scanning. The therapeutic activity of dopamine blockers could then be rationalised as resulting from their ability to repress cytochrome oxidase and restore the pre-pathological situation. These, however, are only speculations and further work is needed to identify and characterise many elements in the chain of cause and effect.

\section{References}

BetTINI, E. \& MAGGI, A. (1992) Estrogen induction of cytochrome c oxidase subunit III in rat hypocampus. Journal of Neurochemistry, 58, 1923-1929.

Buchsbaum, M. S., Nuechterlein, K. H., Haier, R. J., et al (1990) Glucose metabolic rate in normal and schizophrenics during the continuous performance test assessed by positron emission tomography. British Journal of Psychiatry, 156, 216-227.

Chiodo, L. A. \& Bunney, B. S. (1983) Typical and atypical neuroleptics: differential effects of chronic administration on the activity of A9 and A10 midbrain dopaminergic neurons. Journal of Neuroscience, 3, 1607-1619.

Cohen, R. M., Semple, W. E., Gross, M., et al (1987) Dysfunction in a prefrontal substrate of sustained attention in schizophrenia. Life Sciences, 20, 2032-2039.

, - , et al (1988) From syndrome to illness: delineating the pathophysiology of schizophrenia with PET. Life Sciences, 43, 1140-1150. 
COON, H., Byerley, W., Holik, J., et al (1993) Linkage analysis of schizophrenia with five dopamine receptor genes in nine pedigrees. American Journal of Human Genetics, 52, 327-334.

Franzen, G. \& IngVar, D. H. (1975) Abnormal distribution of cerebral activity in chronic schizophrenia. Journal of Psychiatric Research, 12, 195-214.

Gejman, P. V., Ram, A., Gelernter, J., et al (1994) No structural mutation in the dopamine D2 receptor gene in alcoholism or schizophrenia. Analysis using denaturing gradient gel electrophoresis. Journal of the American Medical Association, 271, 204-208.

Hafner, H., Behrens, S., De-Vry, J., et al (1991) An animal model for the effects of oestradiol on dopamine mediated behaviour: implications for sex differences in schizophrenia. Psychiatry Research, 38, 125-134.

Johnstone, E. C., Crow, T. J., Frith, C. D., et al (1978) Mechanism of the antipsychotic effect in the treatment of acute schizophrenia. Lancet, $i, 848-851$.

MuLCRONE, J. \& WhATLY, S. A. (1993) The application of differential cloning techniques in post-mortem studies of neuropsychiatric disorders: a way ahead. Psychological Medicine, 23, 825-829.

- - FerRIER, I. N., et al (1995) A study of altered gene expression in frontal cortex from schizophrenic patients using differential screening. Schizophrenia Research, 14, 203-212.

Nacqui, A., Chance, B. \& Cadenas, E. (1986) Reactive oxygen intermediates in biochemistry. Annual Review of Biochemistry, 55, 137-166.

RoTH, Z., ARCov, Z. \& MARIS, J. (1989) Effects of ovariectomy on energy metabolism in exercising rat muscle studied by ${ }^{31} \mathrm{P}$ NMR. Journal of Applied Physiology, 67, 2060-2065.

Schneebaum, A. B., Sincleton, J. D., West, S. G., et al (1991) Association of psychiatric manifestations with antibodies to ribosomal P proteins in systemic lupus erythematosus. American Journal of Medicine, 90, 54-62.

SCHORK, N. J. \& Guo, S.-W. (1993) Pedigree models for complex human traits involving the human genome. American Journal of Human Genetics, 53, 1320-1337.

Sunhara, R. K., Seeman, P., Van Toll, H. H. M., et al (1993) Dopamine receptors and antipsychotic drug response. British Journal of Psychiatry, 163, 31-38.

Su, Y., BurKe, J., O’NeIL, F. A., et al (1993) Exclusion of linkage between schizophrenia and the D2 dopamine receptor gene region of chromosome $11 \mathrm{q}$ in 112 Irish multiplex families. Archives of General Psychiatry, 50, 205-211.

Szechtman, H., Nahmias, C., Garnett, S., et al (1988) Effect of neuroleptics on altered cerebral glucose metabolism in schizophrenia. Archives of General Psychiatry, 45, 523-532.

TAKAHASHI, Y. (1954) An enzymological study on brain tissue of schizophrenic patients. Carbohydrate metabolism. Folia Psychiatrica Neurologia Japonica, 7, 214-237.

Van-Itallie, C. M. \& Dannies, P. S. (1988) Estrogen induces accumulation of the mitochondrial ribonucleic acid for subunit II of cytochrome oxidase in pituitary tumour cells. Molecular Endocrinology, 2, 332-337.

WALLACE, D. C. (1992) Diseases of the mitochondrial DNA. Annual Review of Biochemistry, 61, 1175-1212.

Wolyniec, P. S., Pulver, A. E., McGrath, J. A., et al (1992) Schizophrenia: gender and familial risk. Journal of Psychiatric Research, 1, 17-27.

Yamazaki, M., Igarashi, H., Hamamoto, M., et al (1991) A case of mitochondrial encephalomyopathy with schizophrenic psychosis, dementia and neuroleptic malignant syndrome. Rinsho-Shinkeigaku, 31, 1219-1223.

R. M. Marchbanks, DSc, J. Mulcrone, PhD, S. A. Whatley, PhD, Department of Neuroscience, Institute of Psychiatry

Correspondence: R. M. Marchbanks, Department of Neuroscience, Institute of Psychiatry, London SE5 8AF

(First received 3 October 1994, final revision 16 January 1995, accepted 7 February 1995) 